\title{
MEASUREMENTS ON THE FREQUENCY EFFECTS AND COUPLING OF SLOTS AND IRISES FOR THE APT CCDTL*
}

\author{
H. Bluem ${ }^{\dagger}$, M. D. Cole, Advanced Energy Systems, Princeton, NJ \\ J. H. Billen, L. M. Young, LANL, Los Alamos, NM \\ P. Greninger, General Atomics, Los Alamos, NM
}

\begin{abstract}
In preparation for the fabrication of the coupled-cavity drift tube linac[1] portion of the Low-Energy Demonstration Accelerator[2] for the Accelerator Production of Tritium[3] project, the effects of coupling slots/irises have been measured and compared to theoretical calculations. A number of multi-cavity cold models have been constructed corresponding to various sections of the coupled-cavity drift tube linac. These models have been used to measure the average cavity to cavity coupling, the frequency effects of the cavity to cavity coupling slots, the coupling into the cavity structures from a waveguide through an iris, and the effect of the iris on the frequency of the cavity being driven. The results of these measurements have been compared to the theoretical values. The effect of a shorting plate placed in one of the feed waveguides was also investigated. This was used to approximate the position of a closed vacuum valve which would be used in the event of a vacuum window failure.
\end{abstract}

\section{INTRODUCTION}

In preparation for the fabrication of the coupled-cavity drift tube linac (CCDTL)[1] portion of the Low-Energy Demonstration Accelerator (LEDA)[2] for the Accelerator Production of Tritium (APT)[3] project, the effects of coupling slots/irises have been measured and compared to theoretical calculations. The LEDA CCDTL accelerates a $100 \mathrm{~mA} \mathrm{CW}$ proton beam from 6.7 MeV to $10.05 \mathrm{MeV}$. The structure contains 48 two-gap accelerating cavities, two types of coupling cavities, and seven bridge couplers. The power from a single klystron will be split four ways to drive four of the bridge couplers through appropriately sized irises. In later sections of the APT accelerator, the drive irises will be placed in the accelerating cavities.

To efficiently fabricate a large accelerator, it is important that the initial machining of the cavities be as close as possible to the desired final configuration without overshooting. In order to accomplish this, one must be able to predict the frequencies of the individual cells, the cell to cell coupling, and the feed iris coupling. To first order, the frequencies of the cells can be predicted using SUPERFISH[4], but without accounting for the effects of the coupling slots, the resultant fabricated cavity will be too low in frequency. If the coupling can be predicted accurately, then the coupling slots between cavities can be fully cut during the initial machining and will not have to be touched during the tuning procedure. This could potentially save a substantial amount of time. The goal of this work was to develop and verify a method of predicting these effects.

A number of multi-cavity cold models have been constructed corresponding to various sections of the CCDTL. These models have been used to measure the average cavity to cavity coupling using the program DISPER[5], the frequency effects of the cavity to cavity coupling slots, the coupling into the cavity structures from a waveguide through an iris, and the effect of the iris on the frequency of the cavity being driven. The results of these measurements have been compared to formulas for the coupling and frequency effects based upon those derived by J. Gao[6],[7]. The amount of agreement between calculated and measured was mixed, with the largest disagreement occurring in the numbers for the coupling between the waveguide and cavity structure.

The effect of a shorting plate placed in one of the feed waveguides was also investigated. This was used to approximate the position of a closed vacuum valve which would be used in the event of a vacuum window failure. The positions of zero frequency effect on the cavity structure and minimum power loss on the shorting plate were found. Appropriate positioning of the vacuum valve will properly terminate the unusable port from the cavity point of view, thus allowing the accelerator to continue operation even in the event of a vacuum window failure, although driving only three of the four ports.

In the next section, the cavity to cavity coupling measurements will be discussed along with the frequency effects of the cavity to cavity slots. In Section 3, the waveguide to cavity coupling measurements will be discussed and compared to the theory. The shorting plate will be covered in Section 4.

\section{CAVITY TO CAVITY COUPLING}

The coupling between cavities has been measured for a number of cold models corresponding to different locations in LEDA. These measurements have provided both the cavity to cavity coupling and the frequency effect of the slots.

The frequency effects have been measured by starting with an accelerating cavity which has no coupling slots.

\footnotetext{
* Work supported by the US DOE under the APT LEDA program.

† Email: bluem@grump.com
} 
The slots were then opened in steps until a little past the point where the desired coupling was reached. The coupling at each step was also derived. The average coupling value was arrived at by measuring the frequencies of the structure resonances. These frequencies were used to calculate the nearest neighbor coupling.

The numbers so obtained have been compared to values calculated using the following formulas.

$$
\begin{aligned}
& k_{m}=\frac{\pi}{3} \mu_{0} e_{0}^{2} L^{3} \frac{H_{a c} H_{c c}}{\left(K\left(e_{0}\right)-E\left(e_{0}\right)\right)} \frac{1}{\sqrt{U_{a c} U_{c c}}} e^{-\alpha_{H} t}, \\
& k_{e}=-\frac{\pi}{3} \varepsilon_{0}(w / L)^{2} L^{3} \frac{E_{a c} E_{c c}}{\left(E\left(e_{0}\right)\right)} \frac{1}{\sqrt{U_{a c} U_{c c}}} e^{-\alpha_{E} t},
\end{aligned}
$$

and

$$
\delta f_{a c}=\frac{f}{4}\left(k_{m} \frac{H_{a c}}{H_{c c}}+k_{e} \frac{E_{a c}}{E_{c c}}\right) \sqrt{\frac{U_{c c}}{U_{a c}}},
$$

where $\mathrm{k}_{\mathrm{m}}$ and $\mathrm{k}_{\mathrm{e}}$ are the magnetic and electric coupling respectively, $\mu_{0}$ and $\varepsilon_{0}$ are the permeability and permittivity of free space, $e_{0}=\left(1-w^{2} / L^{2}\right)^{1 / 2}$, 'w' is the slot half-width, ' $L$ ' is the slot half-length, $H_{a c}, E_{a c}, H_{c c}$ and $E_{c c}$ are the magnetic and electric fields at the slot position in the accelerating and coupling cavities, $\mathrm{K}\left(\mathrm{e}_{0}\right)$ and $\mathrm{E}\left(\mathrm{e}_{0}\right)$ are complete elliptic integrals of the first and second kind, $\mathrm{U}_{\mathrm{ac}}$ and $\mathrm{U}_{\mathrm{cc}}$ are the stored energy in the accelerating and coupling cavities, $\alpha_{\mathrm{H}}$ and $\alpha_{\mathrm{E}}$ are the TM and TE cutoff waveguide decay factors of the slot, ' $t$ ' is the thickness of the slot, $\delta f_{a c}$ is the frequency change in the accelerating cavity due to the slot and ' $\mathrm{f}$ ' is the initial frequency of the cavity before slots are introduced. If $\mathrm{cc}$ is substituted for ac (and vice verse) in the $\delta$ f equation, one obtains the frequency change in the coupling cavity. These equations are derived assuming an elliptical slot, and the field values which are intended to be used are at the center of the slot. In the LEDA CCDTL, the slots are not elliptical in shape. They are approximately a semi-ellipse but with very rounded corners at the equator. We have not attempted to fit the actual shape to an equivalent ellipse. As a result of the odd shape, the slot length and field strength to be used in the formulae are somewhat ambiguous. There is insufficient space in the present paper to describe how the slot length is chosen and from which location the field strength is taken.

Figures 1 and 2 show a comparison between the measurements performed on one of the cold models and the anticipated values as calculated from Eqs. 1, 2 and 3. The coupling factor shows very close agreement except for the first point. The first point represents a "knife-edge" slot of zero thickness. In reality, this slot has some finite thickness which was not accounted for in the calculations. The target coupling in the design is 5\%. On all of the cold models, the prediction of the coupling factor has been very good. On the other hand, as can be seen in Fig. 2, the prediction of the frequency effect of the slot is not quite as good. The discrepancy is manageable, but it is preferable to have better agreement. A large portion of the discrepancy is probably due to the choice of field strength. With a different choice of field strength, the predicted frequency effect can be brought to within a few percent of the measured values while still keeping the coupling factor values within a percent or two.

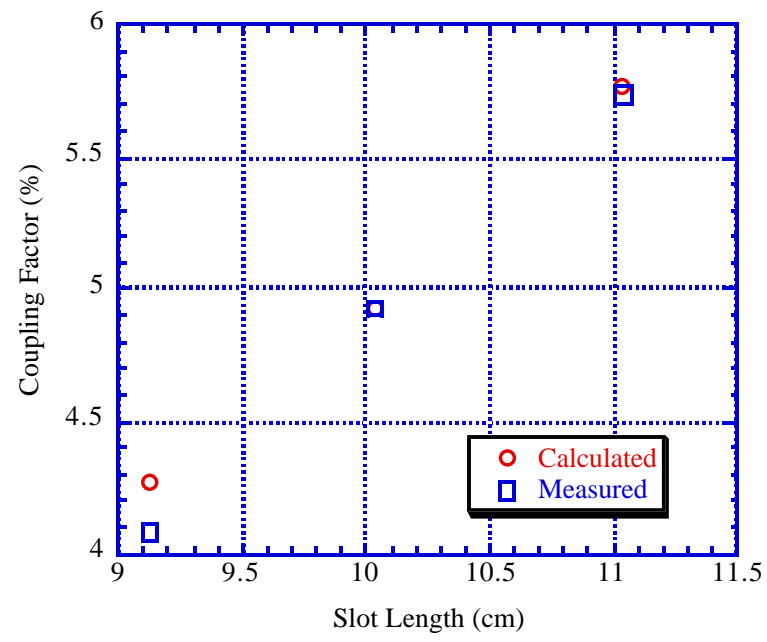

Figure 1. A comparison of the calculated and measured coupling factor as a function of slot length for one of the cold models.

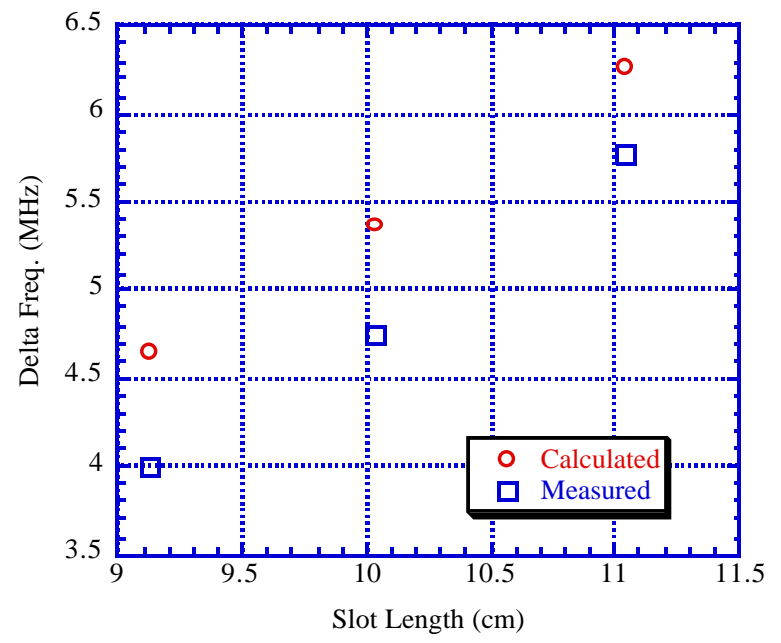

Figure 2. A comparison of the calculated and measured frequency change, in an accelerating cavity, due to the slot as a function of length.

\section{WAVEGUIDE TO CAVITY IRIS}

The waveguide to cavity coupling has been treated in a manner similar to the cavity to cavity coupling. Except in this case, the coupling factor can be measured directly in an easy and straight forward way. The effects of the waveguide input iris have been measured on three different models. The results between the three models have been similar. The equations used to predict the coupling and frequency effect are: 


$$
\begin{aligned}
& \beta=\frac{120 \pi}{9 a b} \sqrt{1-\left(\lambda / \lambda_{c}\right)^{2}} \times \\
& \quad\left[\frac{2 \pi}{\lambda} \pi e_{o}^{2} \frac{L^{x}}{\left(K\left(\mathrm{e}_{o}\right)-E\left(\mathrm{e}_{o}\right)\right)} H_{\text {iris }}\right]^{2} \mathrm{e}^{-2 \alpha t} \frac{Q_{0}}{\omega U_{\text {tot }}}
\end{aligned}
$$

and

$\delta f_{c a v}=-f \frac{\pi}{12} \mu_{0} \frac{L^{3} e_{0}^{2}}{\left[K\left(e_{0}\right)-E\left(e_{0}\right)\right]} \frac{H_{\text {iris }}^{2}}{U_{c a v}}$,

where $\beta$ is the input coupling factor, 'a' and ' $b$ ' are the width and height of the feed waveguide at the iris, $\lambda$ is the free space wavelength of the input signal, $\lambda_{c}$ is the waveguide cutoff wavelength, $x$ is nominally $3, H_{\text {iris }}$ is the field at the iris, $\mathrm{Q}_{0}$ is the unloaded quality factor for the accelerating structure, $U_{\text {tot }}$ is the total stored energy in the accelerating structure, $U_{c a v}$ is the stored energy in the cavity containing the iris, and all others are as before.

The measurements and predicted values of the input coupling and the frequency change as a function of iris length are shown together in Figure 3 for one of the cold models. The predicted coupling was adjusted to fit to the experimental data by varying ' $x$ ' in Eq. 4 . The best fit was given by $x=3.115$.

Since the iris has a relatively large aspect ratio and is generally rectangular in shape, it was easier in this case to generate an equivalent ellipse for the calculations performed. The equivalent ellipse possessed the same length as the iris. The width was increased in order to achieve equal areas between the equivalent ellipse and the actual slot.

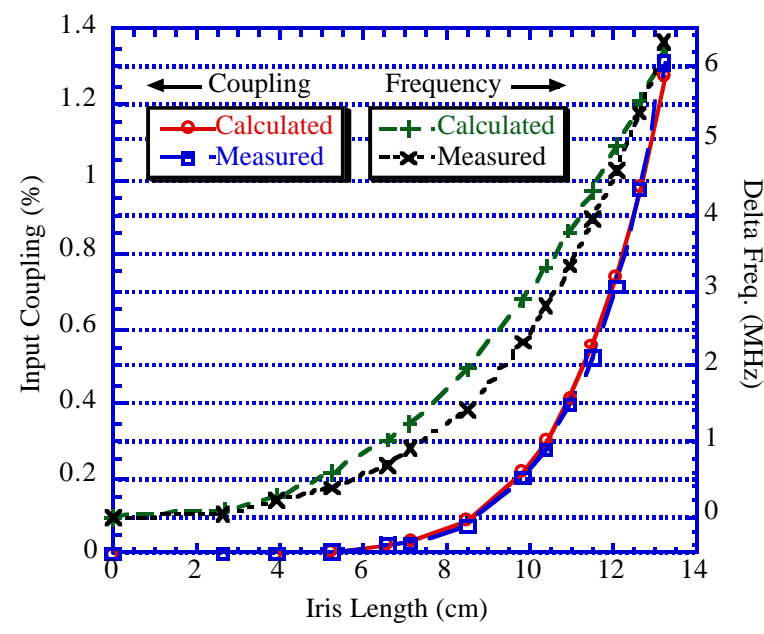

Figure 3. Measured and predicted input coupling and frequency effects as a function of the input iris length.

\section{VALVE/SHORT POSITION}

Due to the desire to keep operating in the event of an RF vacuum window failure, a vacuum valve needs to be placed between the vacuum window and the input iris. This valve, when closed, will present an RF short circuit in the iris feed arm. If this short circuit is appropriately placed, it will have negligible effect on the structure frequency and will have a minimal amount of dissipated energy on its surface. If it is inappropriately placed, it will have a significant effect on the frequency and will have substantial power dissipated on its surface.

Conventional impedance matching theory places the short circuit one quarter of a guide wavelength (or an odd multiple thereof) from the cavity's virtual short position. If the short is placed at such a position, the cavity will not 'see' it.

The measurements determined the cavity's virtual short position to be about $2.54 \mathrm{~cm}$ inside the waveguide measured from the outer surface of the iris. The position proved to be insensitive to the size of the iris. If the short is placed one quarter of a guide wavelength from this point there will be no stored energy in the waveguide arm and hence the power dissipation will be minimized. However, this is not the point of zero frequency effect. The point of zero frequency effect occurs about $1.25 \mathrm{~cm}$ farther back into the waveguide. The position of this point is also insensitive to iris size over a coupling range from about 0.5 to about 2.0.

Since zero frequency effect is most important to proper accelerator operation, the valve will be place at this position. Fortunately, the power dissipation changes very slowly between the minimum power point and this position so that it remains acceptably low. The tolerance on the valve position was found to be plus/minus 2.54 $\mathrm{cm}$ in order to keep the resonant frequency within $15 \mathrm{kHz}$ of nominal.

\section{SUMMARY}

Due to the good agreement between the measured and calculated coupling, the slot sizes can be included in the accelerator design calculations. At this point, accurate incorporation of the frequency effects will require use of an emperically determined factor with the formula.

\section{REFERENCES}

[1] J.H. Billen, et al, "A New RF Structure for Intermediate-Velocity Particles", Proc. 1994 Int. Linac Conf., Tsukuba, Japan, p341 (1994).

[2] H.V. Smith, "Status Update for the Low-Energy Demonstration Accelerator (LEDA)," Proc. 1998 Int. Linac Conf., Chicago, USA.

[3] G. Lawrence, "High Power Proton Linac for APT; Status of Design and Development", Proc. 1998 Int. Linac Conf., Chicago, USA.

[4] J.H. Billen and L.M. Young, POISSON SUPERFISH, Los Alamos National Lab report LA-UR-1834 (revised November 14, 1998).

[5] DISPER coupled cavity modal analysis program maintained and distributed by Los Alamos Accelerator Code Group.

[6] J. Gao, "Analytical formula for the coupling coefficient $\beta$ of a cavity-waveguide coupling system", Nucl. Instr. and Meth. A309, (1991) 5 .

[7] J. Gao, "Analytical formulas for the resonant frequency changes due to opening apertures on cavity walls," Nucl. Instr. and Meth. A311, (1992) 437. 